\title{
Satisfacción de los pacientes hospitalizados con la atención de enfermería
}

\author{
Alma Elizabeth Álvarez de la Rosa,* Clara Olivares Ramírez,** Julio César Cadena Estrada, **** \\ Sandra Sonali Olvera Arreola****
}

\begin{abstract}
RESUMEN
Introducción: La satisfacción en la asistencia de enfermería es entendida como el resultado de la interacción entre las percepciones y las expectativas del paciente constituidas por las vivencias, actitudes y creencias personales. Objetivo: Comparar el grado de satisfacción de los pacientes con la atención de enfermería de acuerdo al género y su asociación con los datos sociodemográficos. Material y métodos: Estudio comparativo, correlacional, prospectivo y transversal. Muestra $(\mathrm{n}=286)$ aleatoria simple tomada del censo diario de los servicios de hospitalización; incluyó pacientes adultos, todos alerta en sus tres esferas y que desearon participar voluntariamente en el estudio. Se utilizó el cuestionario SERVQUAL con una escala de respuesta tipo Likert, dando un valor mínimo de 1 y máximo de 7. Para determinar el grado de satisfacción se utilizó una puntuación: $1-66=$ totalmente insatisfecho, $67-88=$ parcialmente insatisfecho, $89-110=$ regularmente satisfecho, $111-132=$ parcialmente satisfecho y 133-154 = totalmente satisfecho. Datos analizados con el programa SPSS v17 mediante frecuencias, porcentajes, media, prueba de Pearson, Spearman, t de Student y ANOVA; se consideró estadísticamente significativo $p<0.05$. Resultados: El 54.5\% eran hombres; la media de edad fue de $49.9+17$ años. El 57\% refirió estar totalmente satisfecho y el $20.6 \%$ está parcialmente satisfecho con la atención de enfermería; se asocia más con la fiabilidad $(r=0.941, p=0.000)$. Los hombres están más satisfechos que las mujeres $(\mathrm{t}=-1.04, \mathrm{gl}=284, \mathrm{p}=0.295)$; los pacientes con educación superior están más satisfechos. ( $\mathrm{F}$ $=0.580, \mathrm{gl}=284, \mathrm{p}=0.629)$. Conclusiones: La mayoría de los pacientes están satisfechos independientemente de su género, edad o nivel académico; sin embargo, se requiere mantener una atención con calidad y calidez.
\end{abstract}

Palabras clave: Satisfacción, enfermería, calidad.

\section{Hospitalized patients' satisfaction with nursing attention}

\begin{abstract}
Introduction: The satisfaction in nursing care is understood as the result of the interaction between the perceptions and expectations of the patient constituted by the experiences, attitudes and beliefs. Objective: To compare the degree of patient satisfaction with nursing care according to gender and its association with sociodemographic data. Methods: A comparative, correlational, prospective and cross. Sample $(\mathrm{n}=286)$ simple random daily census taken to hospital, included adult patients, warning in three areas and that wish to participate voluntarily in the study. SERVQUAL questionnaire was used a Likert scale, giving a minimum value of 1 and a maximum of 7 . To determine the degree of satisfaction score was
\end{abstract}

\author{
* Jefe de Servicio. \\ ** Adscrita al Servicio de Hospitalización Adultos. \\ *** Subjefe de Educación e Investigación. \\ ***** Jefe del Departamento de Investigación en Enfermería. \\ Instituto Nacional de Cardiología «Ignacio Chávez».
}

Correspondencia: Alma Elizabeth Álvarez de la Rosa. Juan Badiano Núm. 1, Col. Sección XVI, Del. Tlalpan, México, D.F. Tel.: (0155) 5573 2911, Ext. 1391. E-mail: investigacioninc@yahoo.com.mx

Este artículo puede ser consultado en versión completa en http://www.medigraphic.com/enfermerianeurologica 
used: 1-66 = all Satisfied, 67-88 = Mostly dissatisfied, satisfied 89-110 = Regularly, 111-132 = 133-154 = Partly satisfied and completely satisfied. Data analyzed using SPSS v17 as frequencies, percentages, mean, Pearson test, Spearman, and Student $t$ ANOVA was considered statistically significant $\mathrm{p}<0.05$. Results: $54.5 \%$ were male and the mean age was 49.9 \pm 17 years. $57 \%$ reported being completely satisfied and $20.6 \%$ partially satisfacho with nursing care, is more associated with the reliability $(\mathrm{r}=0.941, \mathrm{p}=0.000)$. Men are happier than women $(\mathrm{t}=-1.04$, $\mathrm{df}=284, \mathrm{p}=0.295)$, and patients with higher education are more satisfied. $(\mathrm{F}=0.580, \mathrm{df}=284, \mathrm{p}=0.629)$. Conclusions: Most patients are satisfactory regardless of gender, age or academic level, however, is required to maintain a quality care and warmth.

Key words: satisfaction, nursing, quality.

\section{INTRODUCCIÓN}

$\mathrm{L}$ a calidad de la asistencia sanitaria significa asegurar que cada paciente reciba el conjunto de servicios diagnósticos y terapéuticos más adecuados para conseguir una atención sanitaria óptima, considerando los conocimientos del paciente y del servicio médico, ${ }^{1}$ con lo que se lograrán mejores resultados con el mínimo riesgo y la máxima satisfacción en el proceso administrativo. Así mismo, la calidad asistencial tiene dos componentes: la calidad científico-técnica o intrínseca, que mide la resolución del problema, y la calidad percibida o extrínseca, que mide la satisfacción del usuario con respecto a la percepción del servicio recibido, teniendo en cuenta sus expectativas previas. ${ }^{2}$ Para fines de este estudio, sólo se abordará la calidad extrínseca. La teoría de Donabedian menciona que la calidad está cimentada en estructura, proceso y resultado, lo que constituye hoy en día el paradigma dominante de la evaluación de la calidad de la atención a la salud. ${ }^{2,3}$ Uno de los objetivos del resultado es lograr salud y satisfacción, considerando que si los pacientes se sienten insatisfechos, el cuidado, aunque sea de alta calidad, no será el ideal; ${ }^{4}$ por lo tanto, el valor último de la calidad desde el punto de vista hospitalario es la efectividad del cuidado.

Una institución hospitalaria debe estar basada en un sistema de calidad en servicios de salud, que es el conjunto de elementos y procesos interrelacionados que buscan la satisfacción del usuario, a través del aseguramiento de la calidad y la eficacia operativa de los servicios, todo ello sustentado en una serie de valores que se deben de fomentar en el personal de enfermería, donde se encuentran la actitud, las habilidades, las destrezas, la presencia y el trato. ${ }^{5}$

La mejora de la calidad se puede llevar a cabo a través de la gestión de procesos, cuyo fundamento reside en la visualización del conjunto de actividades, aportando una visión y herramientas con las que se puede actuar, controlar, mejorar o incluso rediseñar el flujo de trabajo para hacerlo eficiente y más adaptado a las necesidades de los clientes a los que va dirigido. Cualquier método o estrategia de garantía de calidad que se aplique serán exitosos mientras el cliente sea el definidor, evaluador, informante, coproductor, ejecutor, controlador y reformador de la calidad. ${ }^{2}$

En el ámbito de la gestión sanitaria se emplean distintos tipos de indicadores para medir la efectividad, entre los que se encuentra la satisfacción de los usuarios, definida como los juicios de valores personales y las reacciones subsecuentes a los estímulos que perciben en la institución hospitalaria, donde evidentemente la satisfacción está influida por las características del paciente, por sus experiencias de salud ${ }^{6}$ y por los hábitos culturales de los diferentes grupos sociales; por tanto, varía según el contexto social. ${ }^{7}$

La opinión del paciente sobre la atención que ha recibido, así como los datos que puede facilitar el proceso, son de gran importancia para cualquier programa de evaluación y mejora de calidad. ${ }^{8}$ El paciente es el evaluador de la calidad asistencial recibida en los servicios sanitarios; estas percepciones subjetivas dan conocimiento de una realidad objetiva; el paciente se convierte en el motor y centro del sistema sanitario, sus necesidades son el eje sobre el que deben articularse las prestaciones asistenciales y constituir la base de la organización de los servicios hospitalarios. El paciente puede evaluar la calidad del servicio sanitario tomando en cuenta no sólo el resultado final, sino también su percepción con la implicación e interés del personal, el trato digno, las instalaciones o el tiempo de atención. En este contexto, la satisfacción del paciente sobre la atención que ha recibido está determinada por factores sociodemográficos y de conocimientos previos sobre el propio concepto de calidad de la atención sanitaria. Los factores o dimensiones que comprende el concepto "satisfacción del paciente" son diversos, y pueden incluir la accesibilidad o disponibilidad de una consulta, las características del hábitat y las facilidades de tipo físico, la confianza en la competencia técnica del personal sanitario, la amabilidad en el trato y la humanización de la asistencia, la información facilitada, la posibilidad de decidir sobre tratamientos, costos y continuidad de los cuidados, la cobertura del sistema de atención sanitaria, el procedimiento burocrático, los resultados de los cuidados, la actitud ante los problemas no médicos, entre otros.

Para fines de la presente investigación se consideraron las siguientes dimensiones: ${ }^{9}$ 
a) La tangibilidad, relacionada con la apariencia de las instalaciones físicas, equipo, personal y material de comunicación. Son los aspectos físicos que el cliente percibe en la organización. Cuestiones tales como limpieza y modernidad son evaluadas en los elementos, personas, infraestructura y objetos.

b) La empatía es la capacidad de brindar cuidado y atención personalizada a sus clientes.

c) La seguridad es el conocimiento que poseen los empleados sobre el quehacer diario, su cortesía y su capacidad de transmitir confianza.

d) La fiabilidad es entendida como la habilidad de desarrollar el servicio prometido precisamente como se pactó y con exactitud.

e) La capacidad de respuesta, como la buena voluntad de ayudar a sus clientes y brindar un servicio rápido.

A pesar de que la calidad es un valor organizacional del sistema de salud y en torno a ella se han incorporado una serie de estrategias para mantenerla y mejorarla de una manera continua a través de la monitorización de indicadores de calidad, se ha observado que existen quejas de los pacientes relacionadas con la atención del personal de salud; por tal motivo, se tiene la finalidad de investigar el grado de satisfacción de los pacientes con la atención de enfermería de acuerdo a algunas variables socioeconómicas como género, edad, nivel académico y tiempo de hospitalización.

\section{MATERIAL Y MÉTODOS}

Se realizó un estudio comparativo, transversal y prospectivo de enero a abril de 2011. La muestra probabilística $(n=286)$ aleatoria se calculó de una población total de 5,400 pacientes con el 30\% de la muestra con el 5\% de error y $95 \%$ de confianza; incluyó pacientes adultos de ambos sexos, que desearon participar voluntariamente, previo consentimiento informado de los servicios de hospitalización; se excluyeron los pacientes de unidades de cuidados críticos.

Se utilizó el cuestionario denominado SERVQUAL con una confiabilidad por Alfa de Cronbach $>0.90 ;{ }^{9}$ constituido por seis preguntas para los datos sociodemográficos y 22 para la satisfacción del paciente respecto a los cuidados deseados y percibidos; distribuido en cinco dimensiones (tangibilidad, fiabilidad, capacidad de respuesta, seguridad y empatía); con una escala de respuesta tipo Likert dando un valor mínimo de 1 y máximo de 7 . El grado de satisfacción se consideró de 1-66 puntos $=$ totalmente insatisfecho, $67-88=$ parcialmente insatisfecho, $89-110=$ regularmente satisfecho, $111-132=$ parcialmente satisfecho y 133-144 = totalmente satisfecho. Para determinar la validez del instrumento en la población mexicana se adaptaron las preguntas al castellano y su aplicación a través de una prueba piloto; se evaluó su confiabilidad interna a través de Alfa de Cronbach y se obtuvo un índice de 0.949. Se analizaron los datos en el programa SPSS versión 17 a través de frecuencias y porcentajes para género, grado académico, estado civil; y media de edad, tiempo de hospitalización y puntuación total de satisfacción y las dimensiones. Así mismo, se realizó análisis inferencial con prueba de Pearson para determinar la asociación entre la puntuación de satisfacción con la edad, tiempo de hospitalización y las dimensiones de fiabilidad, capacidad de respuesta, seguridad y tangibilidad; Spearman para grado de satisfacción con grado académico; t de Student para comparar la satisfacción con género, y ANOVA para grado académico. Se consideró como significativo $\mathrm{p}<0.05$.

\section{RESULTADOS}

La media de edad de los pacientes fue de $49.9 \pm 17$ años con un rango de 18 a 85 años. El $54.5 \%$ eran hombres. Con relación al grado de estudios, el 10\% es analfabeta, $69.9 \%$ tiene estudios de nivel básico y $19.9 \%$ estudios profesionales. De acuerdo al estado civil, el $57.7 \%$ está casado o vive con una pareja y $29.7 \%$ es soltero. El tiempo que llevaban los pacientes hospitalizados fue de 9.26 días en promedio con un rango de 0 hasta 61 días.

En general, el $57 \%$ de la población de estudio está totalmente satisfecho, mientras que el $20.6 \%$ refirió estarlo parcialmente y el $10.8 \%$ regularmente satisfecho. Esto se puede corroborar al identificar que un porcentaje alto da una buena opinión del trato de las enfermeras; y más del $90 \%$ quedó contentó con la atención recibida; esto se refleja en las referencias de los usuarios: darían buena información de los cuidados recibidos, recomendarían el hospital, y en caso de tener en el futuro un problema de salud le gustaría ser atendido otra vez en la institución hospitalaria. Por otra parte, en cuanto al grado de satisfacción de acuerdo al género, el $78.9 \%$ de los hombres está parcial a totalmente satisfecho, mientras que las mujeres sólo el $76.1 \%$ (Cuadro I).

\begin{tabular}{|c|c|c|c|}
\hline \multirow[b]{2}{*}{ Grado de satisfacción } & \multicolumn{2}{|c|}{ Género } & \multirow{2}{*}{$\begin{array}{l}\text { Total } \\
\operatorname{Fr}(\%)\end{array}$} \\
\hline & $\begin{array}{l}\text { Mujer } \\
\text { Fr (\%) }\end{array}$ & $\begin{array}{c}\text { Hombre } \\
\text { Fr (\%) }\end{array}$ & \\
\hline Totalmente insatisfecho & $10(7.7)$ & $7(4.5)$ & $17(5.9)$ \\
\hline Parcialmente insatisfecho & $7(5.4)$ & $9(5.8)$ & $16(5.6)$ \\
\hline Regularmente satisfecho & $14(10.8)$ & $17(10.9)$ & $31(10.8)$ \\
\hline Parcialmente satisfecho & $28(21.5)$ & $31(19.9)$ & $59(20.6)$ \\
\hline Totalmente satisfecho & $71(54.6)$ & $92(59)$ & $163(57)$ \\
\hline Total & $\mathrm{n}=130(100)$ & $n=156(100)$ & $\mathrm{n}=286(100)$ \\
\hline
\end{tabular}


Cuadro II. Comparación de las dimensiones de acuerdo con el género y grado académico.

\begin{tabular}{|c|c|c|c|c|c|c|c|c|}
\hline \multirow[b]{2}{*}{ Dimensiones } & \multicolumn{3}{|c|}{ Género* } & \multicolumn{5}{|c|}{ Grado académico** } \\
\hline & $\begin{array}{l}\text { Hombres } \\
(n=156)\end{array}$ & $\begin{array}{l}\text { Mujeres } \\
(n=130)\end{array}$ & $t$ & $\begin{array}{l}\text { Analfabeta } \\
(n=29)\end{array}$ & $\begin{array}{l}\text { Básico } \\
(n=142)\end{array}$ & $\begin{array}{l}\text { Medio superior } \\
\quad(n=58)\end{array}$ & $\begin{array}{l}\text { Superior } \\
(\mathrm{n}=57)\end{array}$ & $\mathrm{F}$ \\
\hline Tangibilidad & $26.20 \pm 10$ & $27.03 \pm 11.4$ & 0.654 & $25.12 \pm 14.7$ & $27.03 \pm 9.8$ & $26.9 \pm 9.1$ & $25.8 \pm 12$ & 0.371 \\
\hline Fiabilidad & $29.56 \pm 6.9$ & $28.71 \pm 7.7$ & -0.984 & $27.5 \pm 7.2$ & $29.4 \pm 7.5$ & $28.8 \pm 7.5$ & $29.7 \pm 6.3$ & 0.749 \\
\hline Capacidad de respuesta & $23.88 \pm 5.9$ & $23.18 \pm 6.3$ & -0.996 & $22.1 \pm 6.1$ & $24 \pm 6$ & $23 \pm 6.6$ & $23.7 \pm 5$ & 0.339 \\
\hline Seguridad & $23.83 \pm 5.9$ & $23.21 \pm 6.2$ & -0.860 & $22.4 \pm 5.6$ & $23.8 \pm 6.4$ & $23.1 \pm 6$ & $24 \pm 5.6$ & 0.619 \\
\hline Empatía & $28.51 \pm 6.7$ & $27.91 \pm 7.6$ & -0.705 & $27.7 \pm 6.9$ & $27.8 \pm 7.6$ & $28.2 \pm 6.7$ & $29.5 \pm 6.3$ & 0.489 \\
\hline Satisfacción & $129.4 \pm 28.1$ & $125.70 \pm 31.4$ & -1.049 & $122.2 \pm 29.8$ & $18.7 \pm 30.1$ & $125.8 \pm 30.7$ & $130 \pm 25.6$ & 0.580 \\
\hline
\end{tabular}

La puntuación total de satisfacción del paciente hospitalizado está más relacionada con la fiabilidad $(\mathrm{r}=0.941, \mathrm{p}<$ $0.000)$, la seguridad $(\mathrm{r}=0.941, \mathrm{p}<0.000)$, y la capacidad de respuesta $(r=0.939, p<0.000)$, y en menor correlación con la tangibilidad $(r=0.410, p<0.000)$. Estas mismas dimensiones de la satisfacción no tuvieron asociación con la mayoría de los datos demográficos, excepto la tangibilidad con el tiempo de hospitalización $(\mathrm{r}=0.912, \mathrm{p}=0.000)$.

Al comparar la satisfacción de acuerdo al género se encontró que los hombres están más satisfechos con la atención de enfermería al obtener una media más alta que las mujeres $(129.4 \pm 28$ vs $125.7 \pm 31.4)$; sin embargo, esta diferencia no es estadísticamente significativo $(\mathrm{t}=-1.04, \mathrm{gl}=284, \mathrm{p}=$ 0.295 ); de igual forma no fueron significativas las comparaciones entre los pacientes con educación superior y aquellos que tienen estudios de nivel medio superior, básicos o son analfabetas $(\mathrm{F}=0.580, \mathrm{gl}=284, \mathrm{p}=0.629)$ (Cuadro II); y tampoco hubo diferencias significativas entre los días de estancia hospitalaria con la satisfacción del paciente $(\mathrm{F}=$ 4.293, gl 284, $\mathrm{p}=3.82$ ).

\section{DISCUSIÓN Y CONCLUSIONES}

Balseiro y cols. encontraron que los usuarios de un hospital público de México califican de buena a excelente la calidad de la atención proporcionada por las enfermeras y el $72.7 \%$ están satisfechos, ${ }^{10}$ mientras que en Jordania, Mrayyan determinó que los pacientes se encuentran moderadamente satisfechos con el cuidado de enfermería. ${ }^{11}$ Nuestros resultados se asemejan más a lo reportado por Balseiro y cols., a pesar de que las escalas de puntuación no son las mismas; probablemente se deba a la similitud de la población atendida.

En diversos estudios publicados sobre la satisfacción de los pacientes se muestra que entre los factores asociados, la edad se ha relacionado con un mayor grado de satisfacción;;,12 en el estudio de Hernández, los hombres fueron los más satisfechos (94.4\%); así mismo encontró una relación estadística del grado de satisfacción con el tiempo de atención y las instalaciones, ${ }^{13}$ no obstante, en los resultados de este estudio no se encontró una relación con la mayoría de las variables sociodemográficas, excepto la satisfacción con la tangibilidad. En un estudio español, los resultados más relevantes mostraron que la buena comunicación, la información, la empatía, la apariencia de los cuidados y la capacidad técnica son fuertes predictores de la evaluación del cuidado recibido, ${ }^{14}$ variables que se corresponden con las dimensiones del presente estudio, lo que hace notar que la calidad asistencial es percibida de igual forma, independientemente de la región geográfica.

Se coincide con Miraa y cols. ${ }^{15}$ al afirmar que las causas de satisfacción del paciente son la capacidad de respuesta, el confort de las instalaciones (tangibilidad) y la empatía; es de resaltar que esta última variable obtuvo la puntuación más alta en nuestro estudio, lo que demuestra que las expectativas del paciente no se centran únicamente en el tratamiento de la enfermedad; necesitan que se les atienda de manera integral, es decir, que también se perciban sus sentimientos y se aligere su carga emocional derivada de la estancia hospitalaria.

De esta manera, la satisfacción es el resultado de la diferencia entre lo que el paciente esperaba que ocurriera y lo que dice haber obtenido, así como el resultado de la confirmación de las expectativas o de la desconfirmación positiva de éstas ${ }^{16}$ lo que llamamos fiabilidad, circunstancia observada con una fuerte relación estadística en nuestros resultados. Los resultados de esta encuesta son satisfactorios en todas sus dimensiones; no obstante, la fiabilidad es un punto clave que no debemos olvidar, ya que la misión de las instituciones hospitalarias es otorgar atención de calidad y calidez a los usuarios, independientemente de su género, edad o nivel académico. Otra circunstancia a considerar es que los pacientes anteponen la atención personalizada y la capacidad 
de las enfermeras para otorgar cuidados, a las instalaciones físicas, infraestructura o equipo tecnológico.

La atención a los pacientes debe ser integral, y la información referida por los pacientes o sus familiares acerca de su percepción de la calidad de la atención otorgada es una herramienta clave para la implementación de estrategias de mejora continua.

\section{BIBLIOGRAFÍA}

1. Martínez RA. Manual de gestión y mejora de procesos en los servicios de salud. México: Manual Moderno; 2005.

2. García VAM, Soriano CA, Martínez DC, Poblete GVM, Ruiz SS, Cortés RM et al. La satisfacción del usuario como indicador de calidad en un servicio de medicina nuclear. Rev Esp Med Nucl 2007; 26 (3): 146-152.

3. Ortega VMC, Suárez VMG, Jiménez VMC, Añorve GA, Cruz CM, Cruz AG et al. Manual de evaluación del servicio de calidad en enfermería. 2a ed. México: Editorial Médica Panamericana; 2009.

4. Donabedian A. The quality of care. JAMA 1988; 260: 1743-1748.

5. Gutiérrez NA. La calidad en el servicio. Rev Enf IMSS 2004; 12 (1): 1-2.

6. Strasser S, Davis RM. Measuring patient satisfaction for improved patent services. In: Serrano RR, Loriente AN. Anatomía de la satisfacción del paciente. Salud Pública de México. 2008; 50 (2): 161-172. Health Administration Press 1991: 210.

7. Caminal J. La medida de la satisfacción: un instrumento de participación de la población en la mejora de la calidad de los servicios sanitarios. Rev Calidad Asistencial 2001; 16: 276-279.
8. González N, Quintana JM, Bilbao A, Esteban C, San-Sebastian JA, Sierra E et al. Satisfacción de los usuarios de cuatro hospitales del Servicio Vasco de Salud. Gac Sanit 2008; 22 (3): 210-217.

9. Regaria ME, Sola IM, Goñi VR, Del Barrio LM, Margall CMS, Asiain EMC. La calidad asistencial en cuidados intensivos evaluada por los pacientes mediante la escala SERVQUAL. Enferm Intensiva 2010; 21 (4): 136-141.

10. Balseiro L, Martínez P, Ángeles R, Cortés A, De la Rosa M, Flores A et al. La calidad de la atención de Enfermería a los pacientes de un hospital regional del ISSSTE, en México D.F. Un estudio basado en el grado de satisfacción de los usuarios. Revista Universitaria ENEO-UNAM. 2007; (4): 9-13.

11. Mrayyan MT. Jordanian nurses' satisfaction and quality of nursing care. International Nursing Review 2006; (53): PubMed PMID: 16879186.

12. Maldonado G, Medrano MR, Mendiola SV, Nieto MJ, Flores G. El cuidado del adulto mayor. Desarrollo Cientif Enferm 2005; (13): 169189.

13. Hernández MS. Satisfacción de los pacientes sobre la atención de enfermería en el Servicio de Tomografía. Enfer Neurol (Mex) 2010; 9 (3): 155-159.

14. Mascort Z, Pujiula-Masó J, Suñer-Soler R, Puigdemont-Guinart M, Grau-Martín A, Bertrán-Noguer M et al. La satisfacción de los pacientes hospitalizados como indicador de la calidad asistencial. Enferm Clin 2006; 16 (1): 19-26.

15. Miraa JJ, Rodríguez-Marína J, Pesetb R, Ybarrab J, Pérez-Jovera V, Palazóna I et al. Causas de satisfacción y de insatisfacción de los pacientes en hospitales y atención primaria. Rev Calidad Asistencial 2002; 17 (5): 273-283.

16. Rodríguez CE, Ruiz LP, Alcalde EJ, Landa GJ, Villeta PR, Jaurrieta ME. Satisfacción del paciente tras el tratamiento quirúrgico del cáncer colorrectal. Cir Esp 2004; 76 (4): 237-244. 